\title{
Pion-mass dispersion relation in the baryon sector
}

\section{Vladimir Pascalutsa*; Marc Vanderhaeghen}

Institut für Kernphysik, Johannes Gutenberg Universität, Mainz D-55099, Germany

Jonathan M. M. Hall

CSSM, School of Chemistry and Physics, University of Adelaide 5005, Australia

\section{Tim Ledwig}

Departamento de Física Teórica and IFIC, Centro Mixto Universidad de Valencia-CSIC,

E-46071 Valencia, Spain

By looking at the complex plane of the pion-mass squared we establish a dispersion relation which the static quantities, such as baryon masses, magnetic moments, polarizabilities, should obey. This dispersion relation yields insight into the differences between the heavy-baryon and relativistic calculations in the baryon sector of chiral perturbation theory.

7th International Workshop on Chiral Dynamics

August 6 -10, 2012

Jefferson Lab, Newport News, Virginia, USA

*Speaker.

${ }^{\dagger}$ Supported by the Deutsche Forschungsgemeinschaft (DFG) through Collaborative Research Center SFB 1044. 


\section{Introduction}

It is well-known that the chiral perturbation theory $(\chi \mathrm{PT})$ is able to predict some 'non-analytic' dependencies of static quantities (masses, magnetic moments, etc.) on pion-mass squared, or the quark mass $\left(m_{\pi}^{2} \sim m_{q}\right)$. It is therefore interesting to examine the origin of these dependencies arising by considering the analytic properties of chiral expansion in the entire complex $m_{\pi}^{2}$ plane.

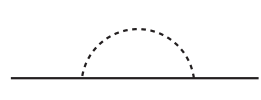

(a)

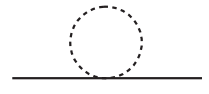

(b)

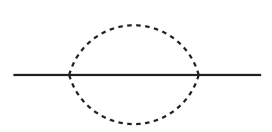

(c)

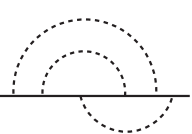

$(d)$

Figure 1: Examples of chiral-loop corrections to the nucleon mass. Nucleon (pion) propagators are denoted by solid (dashed) lines.

Considering the chiral loops with external nucleons on shell, as in the graphs of Fig. 1 arising in the original (manifestly Lorentz-invariant) formulation of baryon $\chi P T(\mathrm{~B} \chi P T)[1]$, we observe that they are analytic functions of $m_{\pi}^{2}$ everywhere except for the branch cut along the negative axis, see Fig. 2. In this case it is possible to write down a simple dispersion relation in the pion-mass squared [2]:

$$
f\left(m_{\pi}^{2}\right)=-\frac{1}{\pi} \int_{-\infty}^{0} \mathrm{~d} t \frac{\operatorname{Im} f(t)}{t-m_{\pi}^{2}+i 0^{+}},
$$

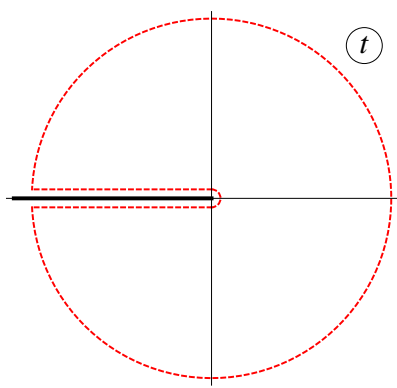

Figure 2: The branch cut and the contour defining the analyticity domain in the complex plane of $t=m_{\pi}^{2}$.

where $f$ is a chiral-loop correction to a static quantity, or the static quantity itself; $0^{+}$is an infinitesimal positive number.

Several applications of this dispersion relation have been discussed in [2]. Here we shall focus on a study of large discrepancies between the leading-order heavy-baryon (HB) $[3,4]$ and $\mathrm{B} \chi \mathrm{PT}[1,5]$ calculations encountered in e.g. Refs. [6-9].

\section{B $\chi$ PT vs. HB $\chi$ PT at $\mathscr{O}\left(p^{3}\right)$}

The chiral expansion of a static quantity $f$ is an expansion in the quark mass $m_{q}$ around the chiral limit $\left(m_{q} \rightarrow 0\right)$, which in $\chi \mathrm{PT}$ becomes an expansion in $p=m_{\pi} / \Lambda_{\chi}$, the mass of the pseudo-Goldstone boson of spontaneous chiral symmetry breaking over the scale of chiral symmetry breaking $\Lambda_{\chi} \simeq 4 \pi f_{\pi} \approx 1 \mathrm{GeV}$. Because of the branch cut in the complex- $m_{\pi}^{2}$ plane along the negative real-axis, the chiral expansion is not a series expansion (otherwise, it would have a zero radius of convergence), but rather an expansion in non-integer powers of $m_{\pi}^{2} \propto m_{q}$.

By writing the dispersion integral as:

$$
f\left(m_{\pi}^{2}\right)=-\frac{1}{\pi}\left(\int_{-\Lambda_{\chi}^{2}}^{0}+\int_{-\infty}^{-\Lambda_{\chi}^{2}}\right) \mathrm{d} t \frac{\operatorname{Im} f(t)}{t-m_{\pi}^{2}},
$$


it is evident that the second integral can be expanded in integer powers of $m_{\pi}^{2} / \Lambda_{\chi}^{2}$. Hence this term is of analytic form and can only affect the values of the LECs. Indeed, the physics above the scale $\Lambda_{\chi}$ is not described by $\chi \mathrm{PT}$ and therefore its effect should be absorbable in the LECs.

The second integral generates an infinite number of analytic terms, while the number of LECs to a given order of the calculation is finite. The higher-order analytic terms are present and not compensated by the LECs at this order, but their effect should not exceed the uncertainty in the calculation due to the neglect of all the other higher-order terms. That is, the second integral can be dropped, while the resulting cutoff-dependence represents the uncertainty due to higher-order effects. We are thus led to examine the cutoff dependence of the pion-mass dispersion relation [10]:

$$
f\left(m_{\pi}^{2} ; \Lambda^{2}\right)=-\frac{1}{\pi} \int_{-\Lambda^{2}}^{0} \mathrm{~d} t \frac{\operatorname{Im} f(t)}{t-m_{\pi}^{2}}\left(\frac{m_{\pi}^{2}}{t}\right)^{n},
$$

where $n$ indicates the number of subtractions around the chiral limit. Our main aim is to see at which values of the cutoff any deviation occurs between the HB- and $\mathrm{B} \chi \mathrm{PT}$ results. If the deviation begins at $\Lambda \ll 1 \mathrm{GeV}$, then the differences between the two expansions cannot be reconciled in a natural way. In the next section, this situation is examined using several specific examples, and for each of them a different picture is obtained (cf. Fig. 3).

At chiral order $p^{3}$, the imaginary parts of the nucleon mass, the proton and neutron AMMs, and the magnetic polarizability of the proton are given by:

$$
\begin{aligned}
\operatorname{Im} M_{N}^{(3)}(t) & =\frac{3 g_{A}^{2} \hat{M}_{N}^{3}}{\left(4 \pi f_{\pi}\right)^{2}} \frac{\pi \tau}{2}\left(\frac{1}{2} \tau+\lambda\right) \theta(-t), \\
\operatorname{Im} \kappa_{p}^{(3)}(t) & =\frac{g_{A}^{2} \hat{M}_{N}^{2}}{\left(4 \pi f_{\pi}\right)^{2}} \frac{2 \pi}{\lambda}\left(\frac{1}{2} \tau+\lambda\right)^{2}\left[1-\frac{3}{2}\left(\frac{1}{2} \tau+\lambda\right)\right] \times \theta(-t), \\
\operatorname{Im} \kappa_{n}^{(3)}(t) & =-\frac{g_{A}^{2} \hat{M}_{N}^{2}}{\left(4 \pi f_{\pi}\right)^{2}} \frac{2 \pi}{\lambda}\left(\frac{1}{2} \tau+\lambda\right)^{2} \theta(-t), \\
\operatorname{Im} \beta_{p}^{(3)}(t) & =-\frac{\left(e^{2} / 4 \pi\right) g_{A}^{2}}{\left(4 \pi f_{\pi}\right)^{2} \hat{M}_{N}} \frac{\pi \tau}{24 \lambda^{3}}[2-72 \lambda+(418 \lambda-246) \tau \\
& \left.-(316 \lambda-471) \tau^{2}+(54 \lambda-212) \tau^{3}+27 \tau^{4}\right] \theta(-t),
\end{aligned}
$$

where $\hat{M}_{N} \simeq 939 \mathrm{MeV}$ is the physical nucleon mass, $e^{2} / 4 \pi \simeq 1 / 137$ is the fine-structure constant, and the following dimensionless variables are introduced:

$$
\tau=\frac{t}{\hat{M}_{N}^{2}}, \quad \lambda=\sqrt{\frac{1}{4} \tau^{2}-\tau} .
$$

The corresponding HB expressions at order $p^{3}$ can be obtained by keeping only the leading in $1 / \hat{M}_{N}$ term (i.e, $\lambda \approx \sqrt{-\tau}$, etc.):

$$
\begin{aligned}
& \operatorname{Im} M_{N}^{(3)}(t) \stackrel{\mathrm{HB}}{=} \frac{3 g_{A}^{2} \hat{M}_{N}^{3}}{\left(4 \pi f_{\pi}\right)^{2}} \frac{\pi \tau}{2} \sqrt{-\tau} \theta(-t), \\
& \operatorname{Im} \kappa_{p}^{(3)}(t) \stackrel{\mathrm{HB}}{=} \frac{g_{A}^{2} \hat{M}_{N}^{2}}{\left(4 \pi f_{\pi}\right)^{2}} 2 \pi \sqrt{-\tau} \theta(-t) \stackrel{\mathrm{HB}}{=}-\operatorname{Im} \kappa_{n}^{(3)}(t), \\
& \operatorname{Im} \beta_{p}^{(3)}(t) \stackrel{\mathrm{HB}}{=} \frac{\left(e^{2} / 4 \pi\right) g_{A}^{2}}{\left(4 \pi f_{\pi}\right)^{2} \hat{M}_{N}} \frac{\pi}{12 \sqrt{-\tau}} \theta(-t) .
\end{aligned}
$$


The full, renormalized result for a given quantity is obtained by substituting these imaginary parts into the dispersion relation of Eq. (2.2). The number of subtractions required in each case differ: $n=2$ for $M_{N}, n=1$ for AMMs, and no subtractions for polarizability. The resulting expressions can be found in [10].

The heavy-baryon expressions can be obtained by picking out the leading in $1 / \hat{M}_{N}$ term, or equivalently, by substituting the corresponding imaginary parts from Eq. (2.5), into the dispersion relation. In the latter case, the same integral is encountered in all of the examples:

$$
J\left(m_{\pi} ; \Lambda\right) \equiv \int_{-\Lambda^{2}}^{0} \mathrm{~d} t \frac{1}{\left(t-m_{\pi}^{2}\right) \sqrt{-t}}=-\frac{2}{m_{\pi}} \arctan \frac{\Lambda}{m_{\pi}} .
$$

All of the above quantities to $\mathscr{O}\left(p^{3}\right)$ in $\mathrm{HB} \chi \mathrm{PT}$ are given by this integral, up to an overall constant, and a factor of $m_{\pi}^{2 n} . n$ is the number of subtractions (or pertinent LECs) at this order. In Fig. 3, the resulting cutoff-dependence of the above loop contributions is shown at the physical value of the pion mass: $m_{\pi} \simeq 139 \mathrm{MeV}$. Each quantity (mass, isovector AMM, and polarizability) is presented

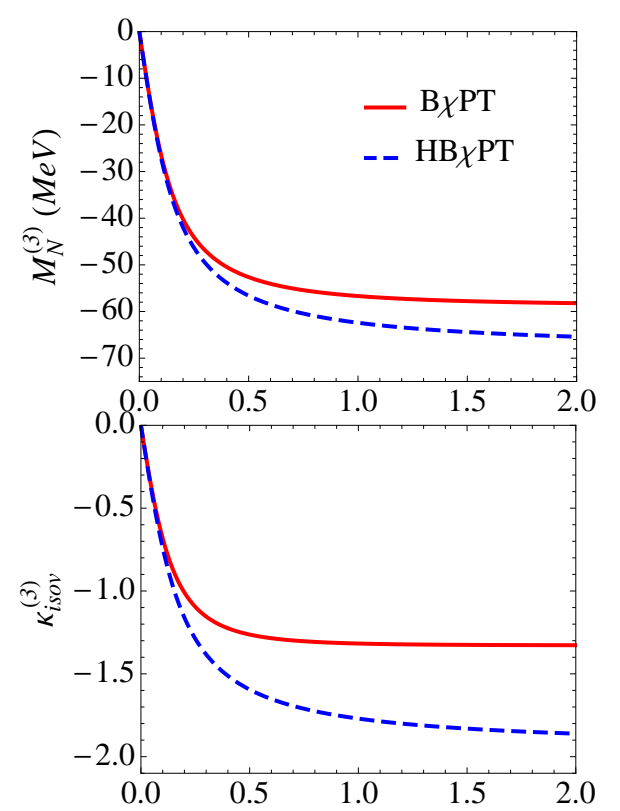

Figure 3: The cutoff-dependence of leading-order loop contributions to various nucleon quantities (mass, isovector AMM, and proton's magnetic polarizability) calculated in $\mathrm{HB} \chi \mathrm{PT}$ (blue dashed curves) and $\mathrm{B} \chi \mathrm{PT}$ (red solid curves).

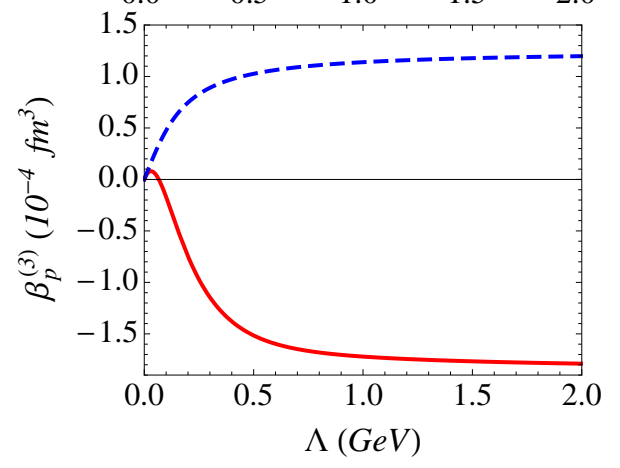


in a separate panel, where the results within the relativistic ChPT and using the HB expansion are displayed.

The figure illustrates the following two features:

1. The $\mathrm{HB} \chi \mathrm{PT}$ results have a stronger cutoff-dependence than the $\mathrm{B} \chi \mathrm{PT}$ results, indicating a larger impact of the unknown high-energy physics to be renormalized by higher-order LECs. Quantitatively, the residual cutoff-dependence in $\mathrm{HB} \chi \mathrm{PT}$ falls as $1 / \Lambda$ in all of the considered examples, while the dependence in the case of $\mathrm{B} \chi \mathrm{PT}$ behaves as $1 / \Lambda^{2}$ for $M_{N}$, and as $1 / \Lambda^{4}$ for both AMMs and $\beta_{p}$.

2. The HB- and $\mathrm{B} \chi \mathrm{PT}$ results are guaranteed to be the same at small values of $\Lambda$, as can be seen by taking derivatives of Eq. (2.2) with respect to $\Lambda^{2}$, at $\Lambda=0$. However, at finite values of $\Lambda$ the differences are appreciable. Observing significant differences for $\Lambda$ of order $m_{\pi}$, as in the case of $\beta_{p}$, indicates that the size of the $1 / \hat{M}_{N}$ terms is largely underestimated in $\mathrm{HB} \chi \mathrm{PT}$.

\section{Conclusion and outlook}

The $\mathrm{HB} \chi \mathrm{PT}$ and $\mathrm{B} \chi \mathrm{PT}$ can be viewed as two different ways of organizing the chiral EFT expansion in the baryon sector. While the heavy-baryon expansion is often considered to be more consistent from the power-counting point of view, it appears to be less natural. Certain terms that are nominally suppressed by powers of $m_{\pi} / M_{N}$, and hence dropped in $\mathrm{HB} \chi \mathrm{PT}$ as being 'higher order', appear to be significant in explicit calculations.

The problem is more pronounced in some quantities and less in others. To quantify this, one needs to note the power of the expansion parameter at which the chiral loops begin to contribute to the quantity in question. For the considered examples of the nucleon mass, AMMs, and polarizability, this power index is 3,1 , and -1 , respectively. The smaller the index, the greater is the difficulty for $\mathrm{HB} \chi \mathrm{PT}$ to describe this quantity in a natural way.

The negative index simply means that the chiral expansion of that quantity begins with negative powers of $m_{\pi}$. Apart from polarizabilities, the most notable quantities of this kind are the coefficients of the effective-range expansion of the nuclear force. The non-relativistic $\chi \mathrm{PT}$ in the two-nucleon sector [11] failed to describe these quantities [12], thus precluding the idea of 'perturbative pions' in this sector. The present work encourages us to think that $\mathrm{B} \chi \mathrm{PT}$ can solve this problem in a way similar to the case of nucleon polarizabilities [8].

\section{References}

[1] J. Gasser, M. E. Sainio and A. Svarc, Nucl. Phys. B 307, 779 (1988).

[2] T. Ledwig, V. Pascalutsa, and M. Vanderhaeghen, Phys. Lett. B 690, 129 (2010);

V. Pascalutsa, PoS CD 09, 095 (2009) [arXiv:0910.3686 [nucl-th]].

[3] E. Jenkins and A. V. Manohar, Phys. Lett. B 255, 558 (1991).

[4] V. Bernard, N. Kaiser, and U. -G. Meissner, Int. J. Mod. Phys. E 4, 193 (1995).

[5] T. Fuchs, J. Gegelia, G. Japaridze, S. Scherer, Phys. Rev. D 68, 056005 (2003). 
[6] V. Pascalutsa, B. R. Holstein, and M. Vanderhaeghen, Phys. Lett. B 600, 239 (2004);

B. R. Holstein, V. Pascalutsa, and M. Vanderhaeghen, Phys. Rev. D 72, 094014 (2005).

[7] L. S. Geng, J. Martin Camalich, L. Alvarez-Ruso, and M. J. Vicente Vacas, Phys. Rev. Lett. 101, 222002 (2008); L. S. Geng, J. Martin Camalich and M. J. Vicente Vacas, Phys. Lett. B 676, 63 (2009).

[8] V. Lensky and V. Pascalutsa, Eur. Phys. J. C 65, 195 (2010).

[9] M. Strikman and C. Weiss, Phys. Rev. C 82, 042201 (2010).

[10] J. M. M. Hall and V. Pascalutsa, Eur. Phys. J. C 72, 2206 (2012).

[11] D. B. Kaplan, M. J. Savage, and M. B. Wise, Nucl. Phys. B 534, 329 (1998).

[12] T. D. Cohen and J. M. Hansen, Phys. Rev. C 59, 13 (1999). 